\title{
The challenge of early detection in cancer
}

\section{Tumour growth dynamics, the timing of metastasis and the limits of cancer screening}

\section{By Nora Pashayan ${ }^{1}$ and Paul D. P. Pharoah ${ }^{2}$}

The chances of survival for a patient with cancer are substantially improved if the disease is diagnosed and treated at an early clinical stage (1). This underpins the promise of early detection to improve prognosis. Longer survival time may reflect later death but it may also reflect advancement of time of diagnosis or increased diagnosis of indolent tumors with no shift in time of death (2). Despite research efforts over several decades, only a handful of early detection tests have been shown to reduce cancerspecific mortality. This benefit comes at a cost: The diagnosis and treatment of cancers that would never have been diagnosed in the lifetime of the patient. Further research is needed to improve cancer early detection methods, but fundamental issues surrounding tumor growth dynamics and when metastases arise make early detection challenging.

An early detection test could be applied in symptomatic patients to reduce the time between presentation and diagnosis. It could also be applied as a 'screening' test in apparently healthy individuals in order to identify those with asymptomatic cancer. It is in the context of screening that early detection is discussed. After malignant transformation, a cancer is small, asymptomatic and undetectable. As it grows it might be detectable by an early detection test before becoming symptomatic and clinically diagnosable. Cancer cells may metastasize at any point, but only a small proportion will grow into macroscopic metastases (3). The majority of deaths from cancer result from widespread metastatic disease (4). Given that cancer mostly occurs in the elderly, mortality from another cause may intervene at any time.

A tumor that has not metastasized at the time of diagnosis can be completely removed and cured by surgery. A tumor that has metastasized may also be curable by a combination of surgery and systemic therapy, but some will be incurable. The probability of achieving systemic cure may depend on the volume of metastatic disease at the time of treatment. The ideal scenario for

${ }^{1}$ Department of Applied Health Research, University College London, London, UK. '2Department of Oncology and Department of Public Health and Primary Care, University of Cambridge, Cambridge, UK. Email:pp10001@medschl.cam.ac.uk early detection is that a tumor that would have metastasized before it is clinically detectable is detected early before the metastasis occurs so the cancer can be cured. An alternative scenario is that detection by screening occurs after the tumor has metastasized but the earlier diagnosis of the metastasis means it is more likely to be curable with systemic treatment.

The potential for screening to detect a tumor before metastasis has occurred depends on factors including growth rate of the tumor, and the relationship between tumor size and metastatic potential (5) (see the figure). Additionally, there is dependence on screening factors including the threshold size at which tumors can be detected and the frequency of screening. The growth rate of tumors of the same tissue of origin in different individuals varies widely, from almost static to fast growing. Most human tumors have a pre-clinical period of at least several years, grow at a constant rate for a prolonged period, and often metastasize before the tumor is clinically detectable (6). A tumor develops its own blood supply once it is $1-2 \mathrm{~mm}$ in diameter so it is assumed that the earliest distant, or bloodborne metastasis can occur is when it is 1 $\mathrm{mm}$ in diameter. The probability of the occurrence of metastasis increases with the size of the tumor at diagnosis $(7,8)$ and fastgrowing tumors are more likely to metastasize than slow-growing tumors $(7,9)$. Screening is less likely to detect the tumors that are most likely to metastasize because they are growing faster.

The impact of tumor growth on the potential for screening can be illustrated for breast cancer because there are good data describing both primary tumor and metastasis growth and because there are data on screening by mammography to detect early disease. The tumor volume doubling time (TVDT) is a measure of the tumor growth rate. In breast cancers it varies from 30 days to more than a year with a median of 150 days $(6,9)$ and metastasis doubling time is usually about half that of the primary tumor (9). If early detection could advance the diagnosis sufficiently the chance of metastasis having occurred at the time of diagnosis would be reduced and the chance of surgical cure increases. Screening would also need to be offered more frequently to detect fast- growing tumors. However, a tumor with a TVDT of 50 days would take just six months to grow from the current limit of detection by mammography ( $\sim 5 \mathrm{~mm}$ in diameter) to a tumor large enough to be diagnosed clinically $(2 \mathrm{~cm})$. It seems unlikely that a population-based screening program with a screening interval of less than one year would be feasible. Therefore, an effective, annual screening test for such tumors would need to be able to detect even smaller tumors. The same tumor would take 16 months to grow from $2 \mathrm{~mm}$ to $2 \mathrm{~cm}$, but currently available technology is not sensitive enough to detect such small lesions.

Detecting a tumor at a smaller size increases the likelihood of detection before metastasis has occurred. However, not all individuals whose cancer is detected earlier by screening benefit from early detection and some are harmed. For example, if metastasis has already occurred at the time of screen detection the metastasis might be too small to be diagnosed, appropriate systemic treatment would not be given, and the chance of cure is reduced. Harm may also arise from an increase in the burden of overdiagnosis and overtreatment. Overdiagnosis refers to the detection of cancers on screening, which would not have become clinically apparent in an individual's lifetime in the absence of screening. Overdiagnosis occurs with detection of either nonprogressive cancer or of slow growing cancer that would take longer than the remaining lifetime of the patient to progress to clinical diagnosis of cancer. Treatment of overdiagnosed cancer does not yield survival benefits but causes emotional and physical harm. If new tests do not distinguish progressive from non-progressive cancers, then early detection will likely increase the incidence of cancer without comparable reduction in cancer-specific mortality.

Detection of smaller tumors could be achieved by screening at shorter screening intervals. Given the heterogeneity of tumor growth rates, more frequent screening would subject a proportion of the population to unnecessary testing and its consequences, including investigation of false test results. Tailoring screening frequency to the tumor growth rates would optimize the benefit-harm trade-off of screening.

The potential of early detection to re- 
duce cancer-specific mortality depends critically on tumor growth rates, metastasis growth rates and the probability that a tumor has metastasized at different points of the growth curve. The relationship of metastasis probability to tumor size has important implications for early detection. Many new imaging technologies are being developed, but it seems unlikely that imaging would be able to detect very small tumors, and so the potential for imaging as a modality for early detection will depend on the probability of early metastasis for different cancers. Historical studies with longterm follow-up of the occurrence of distant metastasis in different types of cancer patients treated by primary surgery without systemic therapy would provide such data. Incorporating such data into multiscale computational modelling platform that can simulate tumor growth for individualized prediction of tumor growth would inform individualized screening strategies.

Blood-based tests, often called liquid biopsies, which detect circulating tumorderived factors such as circulating tumor cells (CTCs) (10) and circulating tumor DNA (ctDNA) (11), have shown potential in early diagnosis. The sensitivity of these tests to detect small tumors is currently too low for use in cancer screening. This may change with technical improvements, but the underlying biology of ctDNA may still limit its potential for early detection. Tumors release DNA into the bloodstream during apoptosis, but, the avoidance of programmed cell death is a hallmark of cancer and those tumors most likely to metastasize early may be least likely to shed DNA into the circulation. Indeed, preliminary modelling studies suggest that tumor growth dynamics influence ctDNA amounts and that a slower growing tumor is associated with a higher ctDNA burden than a faster growing tumor of the same size (12). Given that growth dynamics are an important factor in determining the likely effectiveness of early detection, the balance between earlier detection by liquid biopsy and over diagnosis and over treatment is difficult to predict. Liquid biopsies may also be limited if they are not cancer-site specific. After an abnormal blood test, the site of origin of cancer needs to be identified and imaging will probably be required prior to further investigation, such as biopsy. Ultimately, determining the effectiveness of liquid biopsies for early detection will depend on empirical studies. Even if the sensitivity of liquid biopsies to detect small primary tumors cannot be improved, their application may prove to be in diagnosing metastasis in small tumors detected by other modalities.

There is heterogeneity in neoplastic cells in a tumor and there is some evidence that only a sub-population of tumor cells are able to initiate metastasis (13). Testing screendetected tumors for potential markers of metastasis-forming cells to decide which tumors to treat could reduce overtreatment and improve prognosis. Computational models that can simulate the change in markers of cancer progression and forecast patient-specific cancer progression trajectories could personalize screening strategies and inform when and in whom to initiate treatment (14).

The benefit-harm trade-off from early detection will be maximized by taking into account the heterogeneity of tumor growth kinetics. Consequently, basic research in typical tumor and metastasis growth patterns of cancers at different sites is required. Other priorities in early detection research are methods to identify the small tumors that have the potential to metastasize early and the development of tests to detect occult metastasis at the time of early diagnosis. The ultimate challenge for early detection is to demonstrate an improvement in outcome. Proxy outcomes, such as demonstrating that the application of an early detection test increases the proportion of early stage tumors in all newly-diagnosed tumors known as stage shift - or demonstrating that screen-detected tumors have a longer survival time after diagnosis, are insufficient. An apparent survival benefit of earlier detection may not necessarily lead to prolongation of life because of lead-time bias and length-time bias. Lead-time bias can occur under two scenarios. The first is when a screen-detected tumor would have been detected clinically before metastasis had occurred and so the earlier detection does not improve the outcome. Alternatively, an incurable metastasis has already occurred at the time the tumor is detected by screening and again the long-term outcome is unaffected. Length time bias is due to the preferential detection of slower growing tumors by screening (15). Therefore, randomized controlled trials are needed to establish improvement in health outcomes of different early detection modalities. Clinical trials are expensive and take many years to complete and, given the number of early detection tests being developed, it will not be possible to carry out trials for all of them. Perhaps the most important need is for the research community to establish a strategy for rapidly triaging new tests that are least likely to be effective in order to focus research efforts on those with the most potential.

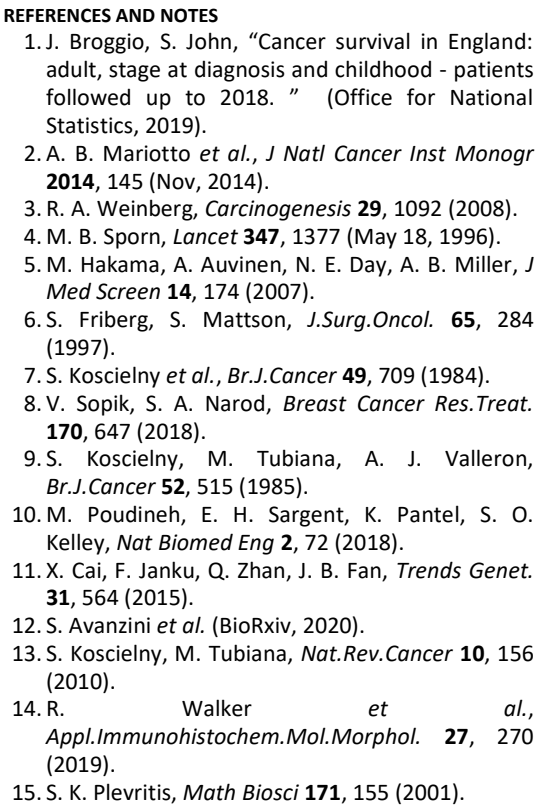

\section{ACKNOWLEDGMENTS}

P. D. P. P. receives salary support from the National Health Service (NHS) in the East of England through the Clinical Academic Reserve.

10.1126/science.aaz2078

FIGURE: Tumor growth dynamics in early detection

Tumor growth dynamics determine whether metastasis is present at the time of currentlyavailable screening tests. Mammographic screening can detect tumors that are $5 \mathrm{~mm}$ in diameter and clinically-detectable tumors are at least $2 \mathrm{~cm}$ in diameter. A typical breast tumor has a tumor volume doubling time (TVDT) of 150 days and will have been growing for 8 years before it is large enough to metastasize ( $>1 \mathrm{~mm}$ ) but it will be another three years before it is big enough to be detected by mammography $(>0.5 \mathrm{~cm})$. The chance that a $0.5 \mathrm{~cm}$ tumor has already metastasized is approximately 5 per cent but the maximum size of that metastasis would be just $0.3 \mathrm{~mm}$. After another 2.5 years the tumor would be $2 \mathrm{~cm}$ with a probability of metastasis of $40 \%$ at which point the maximum size of a metastasis would be 4 $\mathrm{mm}$. Calculations based on method of Friberg and Mattson (6) Based on data from Koscielny et al (7) and Sopik and Narod (8).

A. Probability of lymph node or distant metastasis by primary breast tumor size (distant metastasis solid line; lymph node metastasis - dashed line).

B Log-linear growth of a primary tumour (blue) and metastasis (red) over time. 